\title{
Vivências de um trabalhador de saúde na rede de atenção psicossocial durante pandemia de COVID-19
}

\section{Experiences of a health worker in the psychosocial care network during COVID's pandemic 19}

\author{
Ualison Oliveira Sena ${ }^{1 *}$, Nairan Morais Caldas ${ }^{2}$ Vanessa Thamyris Carvalho dos Santos ${ }^{3}$, \\ Soraya Dantas Santiago dos Anjos ${ }^{4}$ Rozemere Cardoso de Souza ${ }^{4}$
}

\begin{abstract}
RESUMO
Os serviços de saúde mental vêm passando por prejuízos e perdas na garantia da assistência integral aos usuários, por conta da pandemia da COVID-19. Este estudo trata-se de um relato de experiência sobre a vivência e sentimentos de perdas de um educador, técnico e acadêmico de Enfermagem, durante a pandemia da COVID-19, em um Centro de Atenção Psicossocial (CAPS) de um município da região sul do estado da Bahia, Brasil, no ano de 2020. Durante a referida pandemia, observaram-se perdas em trabalhos que vinham sendo realizados no CAPS, por conta do fechamento do serviço, no início, e de pois abertura apenas para consulta médica, produzindo afastamento dos usuários de acompanhamento diário. Essa situação concorreu para agudização de sintomas esperados durante a pandemia, assim como, para situações de emergências psiquiátricas, sentimento de desamparo e morte, implicando em descontinuidade de práticas de cuidado e ressurgimento de processos medicalizantes na atenção psicossocial. A atenção multidisciplinar passou a ser substituída pela centralidade do cuidado na figura do médico e medicações, afetando a promoção da saúde mental. Conclui-se, pela experiência, que se torna emergente a educação permanente das equipes dos CAPS, frente aos desafios e enfrentamento de pandemias, para melhor assistir a todos que vierem a precisar de assistência e que esta seja qualificada e humanizada. Assim, a educação permanente da equipe, pode ser o ponto de partida para resistências e retomada da invenção de novas práticas em saúde mental, que dá ênfase à vida, sem reduzi-la ao diagnóstico ou sintomas.
\end{abstract}

Palavras-chave: Pandemia; COVID-19; Saúde mental; Educação; Serviços Comunitários de Saúde Mental.

\begin{abstract}
Mental health services have been experiencing losses and losses in guaranteeing comprehensive care to users, due to the COVID-19 pandemic. This study is an experience report about the experience and feelings of loss of an educator, technician and academic of Nursing, during the pandemic of COVID-19, in a Psychosocial Care Center (CAPS) of a municipality in the southern region from the state of Bahia, Brazil, in the year 2020. During the referred pandemic, there were losses in works that were being carried out at the CAPS, due to the closure of the service, at the beginning, and then the opening of the service only for medical consultation, producing distance from the users who attended the service. This situation contributed to the exacerbation of symptoms expected during the pandemic, as well as to situations of psychiatric emergencies, feeling of helplessness and death, implying discontinuity of care practices and resurgence of medicalizing processes in psychosocial care. Multidisciplinary care has been replaced by
\end{abstract}

\footnotetext{
${ }^{1}$ Universidade Estadual de Santa Cruz. *E-mail: ualisonsenna@hotmail.com

2 Núcleo Regional de Saúde Sul do estado da Bahia.

${ }^{3}$ Universidade Estadual de Santa Cruz; Universidade do Sudoeste da Bahia.

${ }^{4}$ Universidade Estadual de Santa Cruz.
} 
the centrality of care in the figure of the doctor and medications, affecting the promotion of mental health. It is concluded, through experience, that the permanent education of CAPS teams is emerging, in the face of the challenges and confrontation of the pandemics, to better assist everyone who may need assistance and that it is qualified and humanized. Thus, the permanent education of the team can be the starting point for resistance and the resumption of the invention of new practices in mental health, which emphasizes life, without reducing it to diagnosis or symptoms.

Keywords: Pandemic; COVID-19; Mental Health; Education; Community Mental Health Services.

\section{INTRODUÇÃO}

Desde o advento da Psiquiatria e do hospital psiquiátrico no fim do século XVIII, ainda convivemos no século XXI com uma cultura manicomial, que vê a pessoa com sofrimento mental de forma estereotipada, sendo considerada ameaça por pessoas consideradas "normais". Nesse contexto, o cuidado em saúde mental caracteriza-se por isolamento, exclusão, assistencialismo e medicalização, dentre outros aspectos, implicando perda da autonomia sobre a própria vida e aprisionamento social da pessoa em sofrimento psíquico (FARINHA e BRAGA, 2018).

Por mais de um século, o hospital psiquiátrico também representou fonte de lucro, e, apesar de ter sido considerado uma instituição iatrogênica (AMARANTE 2007), permanece com implicações no processo de exclusão de alguns grupos de pessoas vulneráveis e/ ou marginalizadas, em todos os seguimentos da sociedade. Exemplo disso está no isolamento e internação compulsória de usuários de álcool e drogas em "comunidades terapêuticas", e também no que se veicula nas mídias sociais sobre esse problema de saúde pública, sem que de fato ocorram debates sobre as injustiças sociais que atravessam o tema (BRASIL, 2013).

No Brasil, nos anos 1970 surgiram os primeiros movimentos de mudança da assistência psiquiátrica, que teve na Lei 10216/2001 - conhecida como a Lei da Reforma Psiquiátrica (RP) - o cenário propício para constituição de uma nova forma de compreender e tratar a Saúde Mental. A partir de pensamento crítico e de superação à institucionalização da loucura, por mais de 30 anos vem sendo desenvolvidos serviços de cuidado de base comunitária à pessoa em sofrimento psíquico, a partir de uma "rede denominada "substitutiva" (AMARANTE e NUNES, 2018). 
É importante ressaltar que a Reforma Psiquiátrica é resultado da mobilização de usuários, familiares e trabalhadores da Saúde, com o objetivo de extinguir os manicômios, com consequente criação de serviços descentralizados, que pudessem contemplar outras dimensões da vida das pessoas, como moradia, trabalho, lazer e cultura (AMARANTE e NUNES, 2018). Nesse processo, o termo reabilitação psicossocial surge para definir o cuidado em liberdade, que visa ampliação de trocas sociais, autonomia e exercício de cidadania de pessoas com transtornos mentais (SARACENO, 1998). Disto resultou a implantação de serviços que compõem a Rede de Atenção Psicossocial (RAPS), que teve nos Centros de Atenção Psicossociais (CAPS) uma das principais estratégias de reabilitação psicossocial e de reorientação do modelo da psiquiátrica clássica.

A RAPS integra o Sistema Único de Saúde (SUS) e estabelece os pontos de atenção para o atendimento de pessoas com problemas mentais, incluindo os efeitos nocivos do uso de crack, álcool e outras drogas (BRASIL, 2013). Nos CAPS, os usuários passam a ser acompanhados por equipe multiprofissional, para atendimento pautado no respeito e reabilitação, produzindo viver bem na sociedade, dentro de suas limitações, sendo-lhes garantido os princípios da universalidade, equidade e integralidade.

Nos últimos cinco anos, essa rede passou a ser alvo de mudanças radicais e importantes retrocessos, como o resgate do modelo manicomial e investimentos na internação em hospitais psiquiátricos, implicando em desmonte da política da RP brasileira, implantada ao longo de décadas (AMARANTE e NUNES, 2018). Por outro lado, ressaltam-se os impactos positivos que os novos serviços vêm produzindo na vida dos usuários e seus familiares (AMORIM e OTANI, 2015).

Em 2020, surge a pandemia da COVID-19, causada pelo novo coronavírus (SARS-CoV-2). De acordo com Werneck e Carvalho (2020), a pandemia representa um dos maiores desafios sanitários deste século, com altas taxas de morbimortalidade, em todo o mundo. Desde o seu início, ocorreram incertezas sobre quais seriam as melhores estratégias a serem utilizadas para o enfrentamento da mesma, decorrente do insuficiente conhecimento científico sobre o vírus, da alta velocidade de disseminação e da capacidade de provocar mortes em populações vulneráveis. No Brasil, os desafios foram ainda maiores, devido à desigualdade social, com populações vivendo em 
condições precárias de habitação e saneamento, sem acesso sistemático à água e em situação de aglomeração.

O isolamento social foi recomendado, a fim de reduzir o número de infecções e a mortalidade por conta do novo Coronavírus. Os impactos decorrentes dessa medida afetaram, especialmente, a saúde mental. Contudo, ocorreram perdas nos serviços de atenção psicossocial em todo mundo, tanto para pessoas mentalmente doentes, quanto para usuários de substâncias psicoativas. Segundo a OMS, apenas 7\% dos 130 países que responderam a uma pesquisa, relataram que todos seus serviços de saúde mental continuaram funcionando normalmente, e 93\% relataram serviços limitados devido a vários distúrbios (NEBEHAY, 2020).

De modo semelhante, ocorreram perdas em meu processo de trabalho em um Centro de Atenção Psicossocial (CAPS) de um município do interior do estado da Bahia, Brasil, onde o serviço foi fechado, inicialmente, e depois aberto, mas com suspensão das oficinas terapêuticas da equipe, sem previsão de retorno. Como consequência, constatei implicações, muitas delas, perdas aos usuários e seus familiares e às práticas de cuidado que antes desenvolviam em serviço, que visavam ampliação das trocas sociais nos cenários da vida, autonomia e cidadania dos usuários.

Assim, este trabalho visou relatar a experiência deste autor - educador, técnico e acadêmico de enfermagem - vivida no CAPS e as perdas durante o fechamento e interrupção das práticas desse serviço, em tempos de COVID-19. Pretende contribuir para refletir alternativas de enfrentamento das perdas e de resistência às dificuldades no cotidiano do processo de construção de redes da atenção psicossocial.

\section{METODOLOGIA}

Este trabalho trata-se de um relato de experiência sobre a vivência e sentimentos de perdas de um educador, técnico e acadêmico de Enfermagem, durante a pandemia da COVID-19, em um serviço de saúde mental, CAPS I, de um município da região sul do estado da Bahia, Brasil, no ano de 2020. É também parte integrante de um trabalho de conclusão do curso de Enfermagem de uma instituição de ensino superior pública do referido estado, e contou com a participação de docentes das áreas de saúde mental e saúde coletiva, em todas as etapas do delineamento e desta produção textual. 
O relato de experiência é um texto que descreve precisamente uma dada experiência que possa contribuir de forma relevante para sua área de atuação. É a descrição que um autor ou uma equipe fazem de uma vivência profissional tida como exitosa ou não, mas que contribua com a discussão, a troca e a proposição de ideias para a melhoria do cuidado na saúde. Ele traz as motivações ou metodologias para as ações tomadas na situação e as considerações/impressões que a vivência trouxe àquele (a) que a viveu. O relato é feito de modo contextualizado, com objetividade e aporte teórico. Em outras palavras, não é uma narração emotiva e subjetiva, nem uma mera divagação pessoal e aleatória (UFJF, 2017).

Para embasamento teórico, foi pesquisado nas bases de dados LILACS, SCIELO, PUBMED e GOOGLE ACADÊMICO, trabalhos publicados nesse período, com abordagem para o tema saúde mental e COVID-19, que tivessem relevância para o foco deste relato - impactos da pandemia ao usuário de serviços de saúde mental no âmbito do Sistema Único de Saúde. As leituras contribuíram para discutir os resultados desta experiência.

Os resultados descrevem, portanto, vivências do autor no trabalho em reabilitação psicossocial e seus impactos em um CAPS, o momento da interrupção das práticas e perdas durante pandemia, e reflexões conjuntas sobre futuras ações, frente à necessidade de enfrentamento das perdas e de resistências ao que foi desmontado no serviço.

\section{RESULTADOS}

Passo a descrever vivências do meu cotidiano profissional em um CAPS de uma cidade do litoral sul do estado da Bahia, onde também atuava como técnico de Enfermagem em outro serviço do setor saúde, em tempos de pandemia da COVID-19. Em janeiro de 2018, fui convidado para assumir a função de pedagogo do CAPS, antes da pandemia, e meu trabalho consistia em: fazer atendimentos pedagógicos, coordenar e desenvolver as atividades educativas realizadas diariamente; como muitos dos usuários eram analfabetos, não conheciam números, dinheiro e outros problemas. Assim, a secretaria de saúde do município percebeu a importância de um pedagogo fazer parte da equipe multiprofissional, com a finalidade de tentar resolver ou atenuar esses problemas encontrados. 
. O desafio foi grande, mas, desde o $5^{\circ}$ semestre da graduação em Enfermagem, quando cursei a disciplina Enfermagem em Atenção à Saúde Mental, percebi nas práticas como o trabalho em um centro de Atenção Psicossocial pode ser libertador para a equipe e o usuário equipe, pois aliele torna-se protagonista do cuidado. Passei, então, a perceber o lugar que ocupava como uma experiência ímpar.

Com minha formação de Pedagogo, Técnico em Enfermagem e Acadêmico de Enfermagem pensava que só iria ensinar meus conhecimentos - lembrando os processos lineares da educação. Logo, fui surpreendido, pois no cotidiano de produção de diálogos, no serviço, aprendia muito mais do que ensinava. O usuário do CAPS, que nunca frequentou a escola regular pode e tem toda a capacidade de aprender conhecimentos que o ajude a viver melhor em sociedade. E, mesmo para os que não sabiam ler e escrever, através dos diálogos, foi possível desenvolver uma aprendizagem significativa.

As atividades psicoeducativas desenvolvidas proporcionaram aos usuários o conhecimento sobre letras e números, realização de cálculos, e alguns aprenderam a escrever seu próprio nome. Além dessas atividades, minha participação também era ativa nas oficinas de artes, música, dança, passeios pela cidade e, sempre que possível, nas atividades de lazer. Destaca-se que o município possui lindas praias, utilizadas como espaços para ampliação de trocas sociais, onde a alegria parecia "estampada" no rosto dos usuários do serviço quando esses eram utilizados.

Outra atividade de minha co-participação, que considero de grande relevância, diz respeito à visita domiciliar, pois me permitia ver, dentre outros aspectos do território, como o usuário vive no seu domicilio, sua relação com familiares, vizinhança, e como a família estava instruída quanto à supervisão no uso da medicação que alguns usam em casa. Além disso, atividades culturais eram realizadas sempre, lembrando datas comemorativas, como Carnaval, Páscoa, São João, Primavera, 7 de setembro e Natal.

Assim, a prática pedagógica, contrária ao reducionismo de simples oficinas com algumas folhas de papel e orientadas aos desenhos para pintarem impulsionava o fazer pedagogico, buscando atribuir significados de aprendizagem para a vida dos usuários e era pautadada na reabilitação psicossocial, que constitui um processo de invenção e 
reinvenção contínuas, de superação do modelo manicomial e de ampliação das trocas sociais. Contudo, a pandemia da COVID-19, trouxe grandes perdas em muitos trabalhos que eram realizados no CAPS. No início, no mês de março de 2020, orientado pela medida do isolamento, o serviço foi fechado, e mesmo, tendo seu retorno 5 (cinco) meses depois, as atividades educativas permaneciam suspensas, sendo mantida apenas ações voltadas para o atendimento médico e administração de medicamentos.

A partir da suspensão das referidas oficinas terapêuticas no CAPS e da minha atuação em outros serviços da saúde, de caráter hospitalar, na condição de técnico de Enfermagem, percebi grande dispersão dos usuários, uso irregular das medicações ou não uso das mesmas, abandono do tratamento, situações de crise, manejo inadequado da crise, recaídas ao uso de drogas e uso do serviço de emergência constante, mostrandome um futuro incerto quanto ao risco de mais perdas, relacionados à descontinuidade do trabalho.

Ao sentimento de incerteza, somou-se a angústia pelo cenário de perdas no processo de reabilitação que estava em curso na relação com os usuários. Foi e está sendo muito difícil ver todo o trabalho se perdendo, e, mais ainda, perder pessoas. Exemplo disso, é a história de um usuário que chamaremos aqui de R.S.O., um rapaz que já tinha histórico de envolvimento com drogas ilícitas e que em sua família todos conviviam com algum transtorno mental, porém ele e sua mãe frequentavam o CAPS assiduamente, antes da pandemia. Quando comecei a trabalhar no serviço ele já tinha um tempo sem fazer uso de drogas e era aquela pessoa que todos os profissionais e usuários do CAPS e comunidade conheciam por ter uma boa relação com todos e por conta de seu envolvimento com a igreja, porém, com o isolamento, ele voltou a usar drogas, passou a cometer furtos para uso das mesmas, além de ter dívidas com pessoas do tráfico (segundo relatos de pessoas da cidade).

Nesse contexto, R.S.O. sofreu várias agressões físicas, sendo que da última vez, precisou ser internado para uma laparotomia de emergência no Hospital Regional, que é referência para a cidade. Foi constatado que seu apêndice tinha se rompido, sendo internado pós cirurgia na UTI, mas não resistiu e foi a óbito. O hospital regional ficava a $60 \mathrm{~km}$ do município de origem do paciente, no entanto, ocorreu demora no translado do corpo, que voltou para o município de origem, vinte horas após o óbito. 
No dia da remoção do corpo de R.S.O., eu estava de plantão no hospital do município, e fui informado pela assistente social do serviço que o hospital que ele estava não vestia o corpo e que a funerária que fez o transporte também não e que teríamos que pagar outo valor para vesti-lo. Ele estava apenas de fraldas e a família queria que vestisse para sepultá-lo. Então recebemos o corpo no hospital em totalmente rigidez cadavérica, pois tinha ficado todo esse tempo no refrigerador, e eu que ali estava como Técnico de Enfermagem, fui designado para fazer o preparo do corpo para sepultamento. Isso impactou muito em mim, enquanto profissional e pessoa, pois havia desenvolvido vínculo com ele. Além dos conhecimentos compartilhados e da alfabetização, tinha perdido alguém com quem vínhamos construindo um projeto de felicidade e de vida, e que agora, havia chegado ao fim.

Toda equipe do CAPS e eu ficamos muito entristecidos com a morte de R.S.O. e também com o contexto da pandemia, pois percebemos há uma invisibilidade quanto ao sofrimento psíquico e uma grande lacuna no processo de matriciamento entre os pontos da rede do município, sendo a Estratégia de Saúde da Família (ESF) e o CAPS protagonistas no planejamento para melhora da qualidade de vida de usuários em sofrimento mental.

Como trabalhador da saúde, quero, ainda, registrar que fui infectado pela COVID-19, e durante o isolamento, confesso que também tive sintomas de transtorno de ansiedade, pelo medo de não conseguir viver frente ao número de perdas de pacientes que presenciei enquanto cuidava deles. Mesmo assim, ainda é comum diariamente esse sentimento, pois a pandemia continua no Brasil.

Percebe-se, nesse momento difícil de pandemia que a população mundial está passando que é preciso pensar em novas estratégias para não deixar as pessoas que utilizavam, diariamente, os serviços de saúde mental, sem assistência. E também, para resistir às perdas e aos desmontes do processo de reabilitação psicossocial que tem reduzido os CAPS em unidades ambulatoriais. Assim, passei a refletir a necessidade emergente de capacitação da equipe dos serviços de saúde mental, o que se torna fundamental para retomada dos atendimentos e criação de novas estratégias terapêuticas, em tempos de pandemia. 


\section{DISCUSSÃO}

A COVID-19 atingiu as pessoas em diferentes níveis de complexidade, com impactos na saúde mental. Algumas com história pregressa de transtornos mentais e quadros estáveis acabaram em situação de crise, ocasionando perdas em vários segmentos da vida, outras que não tinham problemas mentais passaram a desenvolver por conta do contexto vivido pela pandemia (BRASIL, 2020).

Além de perdas como as que foram listadas no decorrer da descrição deste relato, pesquisas recentes mostram aumento no número de crises de ansiedade, fobia, transtorno depressivo e pensamentos suicida. A rápida mudança nos modos de vida habituais pode contribuir para o desencadeamento de reações e sintomas de estresse, ansiedade e depressão. Adicionalmente, o medo de ser acometido por uma doença potencialmente fatal, cuja causa e progressão eram pouco conhecidas, afetou o bemestar psicológico das pessoas e também de nós, profissionais da saúde (BRASIL, 2020).

Esse cenário agravou as situações de perdas enfrentadas por serviços de Saúde Mental, quanto às atividades que eram desenvolvidas com os usuários, principalmente, aqueles que frequentavam em regime intensivo, e também afetou a missão do próprio serviço, reduzindo-o à atenção ambulatorial.

Os CAPS, nas suas diferentes modalidades, são pontos de atenção estratégicos da RAPS: serviços de saúde de caráter aberto e comunitário constituídos por equipe multiprofissional que atua sob a ótica interdisciplinar e realiza prioritariamente atendimento às pessoas com sofrimento ou transtorno mental, incluindo aquelas com necessidades decorrentes do uso de crack, álcool e outras drogas, em sua área territorial, sejam em situações de crise ou nos processos de reabilitação psicossocial (BRASIL, 2015).

A composição da equipe multiprofissional no CAPS requer constante processo de formação, a fim de se pensar e desenvolver novas estratégias de enfrentamento, inclusive, durante pandemia, para garantir continuidade das atividades dos serviços, atenuando e superando perdas que vem acontecendo frequentemente.

$\mathrm{Na}$ experiência relatada, vimos que medidas de controle e de prevenção da COVID-19 descritas pela quarentena e isolamento social, fundamentais para evitar a propagação do vírus, afetaram a continuidade do acompanhmento dos usuários. Para 
Faro et al (2020), a quarentena busca separar e restringir a circulação de pessoas que foram expostas a uma doença contagiosa, visando observar a ocorrência de novos casos, e o isolamento diz respeito à separação de pessoas doentes dos não doentes, infectadas por alguma doença transmissível, como a COVID-19.

A partir de medidas de segurança higienização das mãos, uso de máscaras, colocação de recipientes com álcool gel no ambiente, diminuição do tempo de permanência no local, alguns atendimentos poderiam voltar a acontecer com o objetivo de reduzir os danos psicológicos causados pela Pandemia. Contudo, o retorno das atividades no serviço levou um tempo prolongado para acontecer.

Mesmo com a quarentena e o isolamento, outro fator que também dificultou o andamento do serviço no início da pandemia foi a não testagem da população, pois, ainda, a quantidade de testes era insuficiente, para avaliar todas as pessoas, deixando os testes apenas para as pessoas consideradas sintomáticas para a COVID-19. Com realização da testagem, seria possível identificar se a pessoa já contaminou ou não e assim dar continuidade às atividades, respeitando-se as medidas de segurança preconizadas pela Organização Mundial de Saúde (OMS). Ressalto, ainda, que outra estratégia seria a continuidade da visita domiciliar para desenvolver a escuta terapêutica que é uma tecnologia do cuidado.

Assim, era necessário criar meios de mitigação para atenuar e se possível resolver problemas que afetavam os serviços, o que dependia também dos profissionais de saúde, os quais deveriam ter uma visão mais acolhedora e comprometida em ajudar na manutenção desse movimento, para que as pessoas em sofrimento mental fossem assistidas e com dignidade (ABBUD, 2018).

Para isso, é preciso deixar que o foco da assistência em saúde mental seja apenas medicamentosa e biologicista e valorizar os processos psicossociais. Isto não quer dizer que não seja preciso medicar, mas essa não deve ser a única finalidade do serviço "medicar e diagnosticar" sendo esse último, enquadrar a pessoa em determinada classificação de diagnósticos psiquiátricos. Os diagnósticos psiquiátricos aparecem quase sempre com base biológica, aproximando o sofrimento psíquico das doenças orgânicas, colocando-os quase como exclusivos de desordens da bioquímica cerebral, o que também fomenta a indústria farmacêutica, que investe de forma maciça em pesquisas, produção e marketing de novos remédios (ZANELLA et al., 2016). 
Nesse aspecto, a educação permanente da equipe tem papel primordial para frear a medicalização, e fazer com que as equipes mudem suas estratégias de atendimentos e assistência aos usuários, adotando outras formas de cuidado, a saber: escuta terapêutica, oficinas de alfabetização, visitas domiciliares, oficinas de pintura e artesanatos, rodas de conversa, passeios por pontos turísticos do município, fazendo com que os usuários não sejam institucionalizados, mas sim pessoas participantes da sociedade. Para isso, a articulação do NASF, ESF e CAPS é de grande relevância, pois possibilita o fortalecimento de redes de cuidado, onde a Educação Permanente em Saúde é estratégia fundamental de reflexões críticas e atuação comprometida, superando, assim, a hegemonia médica do encaminhamento, concretizando vínculo do usuário com os outros atores da rede, familiares e comunidade (ARCE et al., 2011).

Nesse sentido, os arranjos de formação de equipes de saúde já não têm o profissional médico como prescritor, como protagonista no tratamento dos usuários. Nesse novo modelo, advindo da reforma da atenção psiquiátrica, as equipes são formadas por vários profissionais de nível médio, técnico e superior, e o ator principal passa a ser o usuário e não a equipe. A intervenção deixa de ser apenas médica e passa a ser multiprofissional, sendo o médico apenas integrante da equipe multiprofissional e não tendo grande papel de destaque. $\mathrm{Na}$ equipe todos tem grande relevância para o serviço de atendimento às pessoas em sofrimento psíquico.

Frente aos desafios da pandemia da COVID-19, é preciso manter o desafio permanente da construção de um novo paradigma em saúde mental. Essa proposta ainda continua requerendo o que afirmou Campos e Barros (2000, p.276) sobre o papel do enfermeiro: "rever a formação, apontando para novas posturas frente ao sujeito e seu sofrimento psíquico; ampliar conhecimentos para além dos adquiridos na graduação, compartilhando efetivamente práticas e saberes subjacentes a essas práticas, com outros campos disciplinares". Isto se aplica a todas as profissões e este relato pretende contribuir com esses diálogos e mudanças de posicionamentos.

Assim, o objetivo permanente do serviço de saúde mental e porque não dizer da equipe multiprofissional, será sempre reduzir danos, perdas e proporcionar o desenvolvimento de autonomia para a vida dos usuários em sociedade. E, cada vez mais, se preciso for, se reinventar para as necessidades e desafios encontrados nos serviços, de modo progressivo e crescente, inclusive em tempos de pandemia. 


\section{CONSIDERAÇÕES FINAIS}

Este relato de experiência permitiu refletir sobre a rotina de um serviço em saúde mental, percebendo que este, no momento crítico em que estamos vivendo, precisa ser repensado em sua atuação junto à comunidade, não apenas em relação aos usuários, mas também se preparar para atender toda essa demanda que está surgindo, decorrentes dos impactos causados pela pandemia da COVID-19. Para isso, é preciso priorizar os atendimentos intensivos para o CAPS, e também o acompanhamento dos mesmos pela Estratégia de Saúde da Família, superando a dicotomia da Atenção Primária a Saúde com os serviços da Atenção Psicossocial, pois a pessoa em sofrimento psíquico não deve ser visto como um usuário apenas do CAPS, mas sim de toda Rede de Apoio Psicossocial, que inclui a Estratégia de Saúde da Família como grande protagonista, ordenadora e coordenadora do cuidado, e porta de entrada para o Sistema Único de Saúde (SUS).

É relevante que a rede de apoio, nesse momento, se articule de forma a garantir o atendimento de forma integral aos usuários e também perceber que muitos profissionais de saúde que estão atuando na linha de frente, também estão sendo acometidos por algum transtorno mental e passando a necessitar dessa assistência.

Finalmente, considero que foi difícil a escrita deste trabalho, ao mesmo tempo, considero-o como uma reflexão necessária, pois precisamos evitar que tais perdas continuem acontecendo. Um caminho possível seria o da educação permanente da equipe, frente aos desafios e enfrentamento de pandemias, como tem sido a da COVID19, para melhor acolher a todos que precisam de assistência e que esta seja qualificada e humanizada. Assim, a educação permanente da equipe, pode ser o ponto de partida para retomada e concretização da reabilitação psicossocial e também de apoio ao trabalhador da saúde.

\section{REFERÊNCIAS}

ABBUD, Carolina. EVOLUÇÃO DOS CUIDADOS DE ENFERMAGEM EM

SAÚDE MENTAL. In Enfermagem em saúde mental. Editora: SAGH. Disponível 
em:〈https://sagahcm.sagah.com.br/sagahcm/sagah_ua_dinamica/26743137\#leia> Acesso em 01 de Dez. 2020.

AMARANTE, Paulo. SAÚdE MENTAL E ATENÇÃO PSICOSSOCIAL. Rio de Janeiro: Editora Fiocruz; 2007. 120 pp. (Temas em Saúde).

AMARANTE, Paulo; NUNES, Mônica Oliveira. A reforma psiquiátrica no SUS e a luta por uma sociedade sem manicômios. Ciência \& Saúde Coletiva, v. 23, n.6, p. 20672074, 2018. https://doi.org/10.1590/1413-81232018236.07082018

AMORIM, Marianna de Francisco; OTANI, Márcia Aparecida Padovan. A reabilitação psicossocial nos Centros de Atenção Psicossocial: uma revisão integrativa. SMAD Revista Eletrônica Saúde Mental Álcool e Drogas (Edição em Português), [S. l.], v. 11, n. 3, p. 168-177, 2015. DOI: 10.11606/issn.1806-6976.v11i3p168-177. Disponível em: https://www.revistas.usp.br/smad/article/view/116773. Acesso em: 14 out. 2021.

ARCE, Vladimir Andrei Rodrigues; SOUSA, Maria Fátima de; LIMA, Maria da Glória. A práxis da Saúde Mental no âmbito da Estratégia Saúde da Família: contribuições para a construção de um cuidado integrado. Physis Revista de Saúde Coletiva, Rio de Janeiro, 21 [ 2 ]: 541-560, 2011. Disponível em:

https://www.scielo.br/j/physis/a/FVWCKDXMttCs4bQBp4vxggq/?format=pdf\&lang=p $\mathrm{t}$

BRASIL. Ministério da Saúde. Secretaria de Atenção à Saúde. Departamento de Atenção Básica. Saúde mental / Ministério da Saúde, Secretaria de Atenção à Saúde, Departamento de Ações Programáticas Estratégicas. - Brasília: Ministério da Saúde, 2013. 176 p. (Cadernos de Atenção Básica, n. 34)

BRASIL. SAÚdE MENTAL E ATENÇÃO PSICOSSOCIAL NA PANDEMIA COVID-19. A quarentena na Covid-19: Orientações e estratégia do cuidado. Ministério da Saúde. Fundação Oswaldo Cruz. - Brasília: Ministério da Saúde, 2020.

CAMPOS, Célia.Maria.Sivalli;BARROS, Sônia. Reflexões sobre o.processo de cuidar da enfermagem em saúde mental. Rev.Esc.Enf.USP, v.34, n.3, p. 271-6, set. 2000. https://doi.org/10.1590/S0080-62342000000300008

FARINHA, Marciana Gonçalves; BRAGA, Tatiana Benevides Magalhães. Sistema único de saúde e a reforma psiquiátrica: desafios e perspectivas. Rev. abordagem gestalt., Goiânia , v. 24, n. 3, p. 366-378, dez. 2018 . Disponível em $<$ http://pepsic.bvsalud.org/scielo.php?script=sci_arttext\&pid=S1809$68672018000300009 \& \operatorname{lng}=$ pt\&nrm=iso $>$. acessos em 14 out. 2021. http://dx.doi.org/10.18065/RAG.2018v24n3.11.

FARO, André et al. COVID-19 E SAÚDE MENTAL: A EMERGÊNCIA DO CUIDADO. Estud. psicol. (Campinas), Campinas, v. 37, e200074, 2020. Disponívelem<http://www.scielo.br/scielo.php?script=sci_arttext\&pid=S0103166X202 $0000100507 \& \operatorname{lng}=$ pt\&nrm=iso $>$. acessos em 26 nov. 2020. Epub 01-Jun-2020. http://dx.doi.org/10.1590/1982-0275202037e200074.

NEBEHAY, Stephanie. OMS: SERVIÇOS DE SAÚDE MENTAL DIMINUÍRAM DURANTE PANDEMIA. Organização considera que esse é um aspecto esquecido da covid-19. Disponível em: < https://agenciabrasil.ebc.com.br/internacional/noticia/2020- 
10/oms-servicos-de-saude-mental-diminuiram-durante-pandemia> Acesso em: Dez. 2020.

SARACENO, Benedeto. A concepção de reabilitação psicossocial como referencial para as intervenções terapêuticas em saúde mental. Revista de Terapia Ocupacional da Universidade de São Paulo, São Paulo, v. 9, n. 1, p. 26-31, 1998.

UFJF. INSTRUTIVO PARA ELABORAÇÃO DE RELATO DE EXPERIÊNCIA; Estágio em Nutrição em Saúde Coletiva. 2017. Disponível em:< https://www.ufjf.br/nutricaogv/files/2016/03/Orienta\%C3\%A7\%C3\%B5esElabora\%C3 $\% \mathrm{~A} 7 \% \mathrm{C} 3 \%$ A3o-de-Relato-de-Experi\%C3\%AAncia.pdf $>$ Acesso em: 22 de novembro de 2020.

WERNECK, Guilherme Loureiro; CARVALHO, Marilia Sá. A pandemia de COVID19 no Brasil: crônica de uma crise sanitária anunciada. Cad. Saúde Pública, Rio de Janeiro, v. 36, n. 5, e00068820, 2020 . Available from $<$ http://www.scielo.br/scielo.php?script=sci_arttext\&pid=S0102311X2020000500101\& lng=en\&nrm=iso $>$.accesson 19 Nov. 2020. Epub May 08, 2020. https://doi.org/10.1590/0102-311x00068820.

ZANELLA, Michele et al . Medicalização e saúde mental: Estratégias alternativas. Revista Portuguesa de Enfermagem de Saúde Mental, Porto, n.15, p.5362, jun. 2016.Disponívelem<http://www.scielo.mec.pt/scielo.p $\mathrm{hp}$ ?script=sci_arttext\&pid=S164721602016000100008\&lng=pt\&nrm=iso $>$.acessosem 09 dez. 2020. http://dx.doi.org/10.19131/rpesm.0132.

Recebido em: 01/09/2021

Aprovado em: 25/09/2021

Publicado em: 30/09/2021 